Supporting information

\title{
Above-Room-Temperature Ferroelastic Phase Transitions in Two Tetrafluoroborate-based Hexagonal Molecular Perovskites
}

Xiao-Xian Chen, De-Xuan Liu, Ya-Ping Gong, Sha-Sha Wang, Wei-Xiong Zhang, ${ }^{*}$ and Xiao-Ming Chen

MOE Key Laboratory of Bioinorganic and Synthetic Chemistry, School of Chemistry, Sun Yat-Sen University, Guangzhou 510275, China

*Corresponding author email: zhangwx6@mail.sysu.edu.cn 


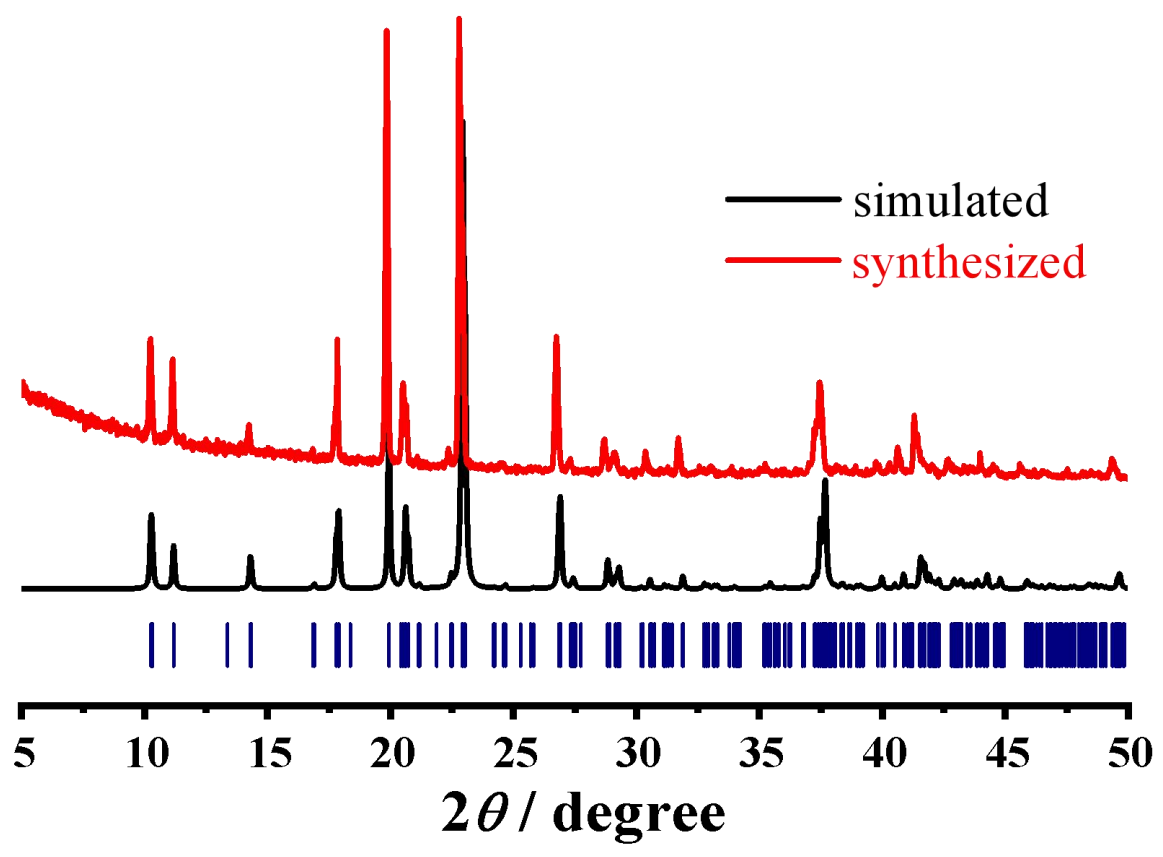

Figure S1. The PXRD patterns of $\mathbf{1 .}$

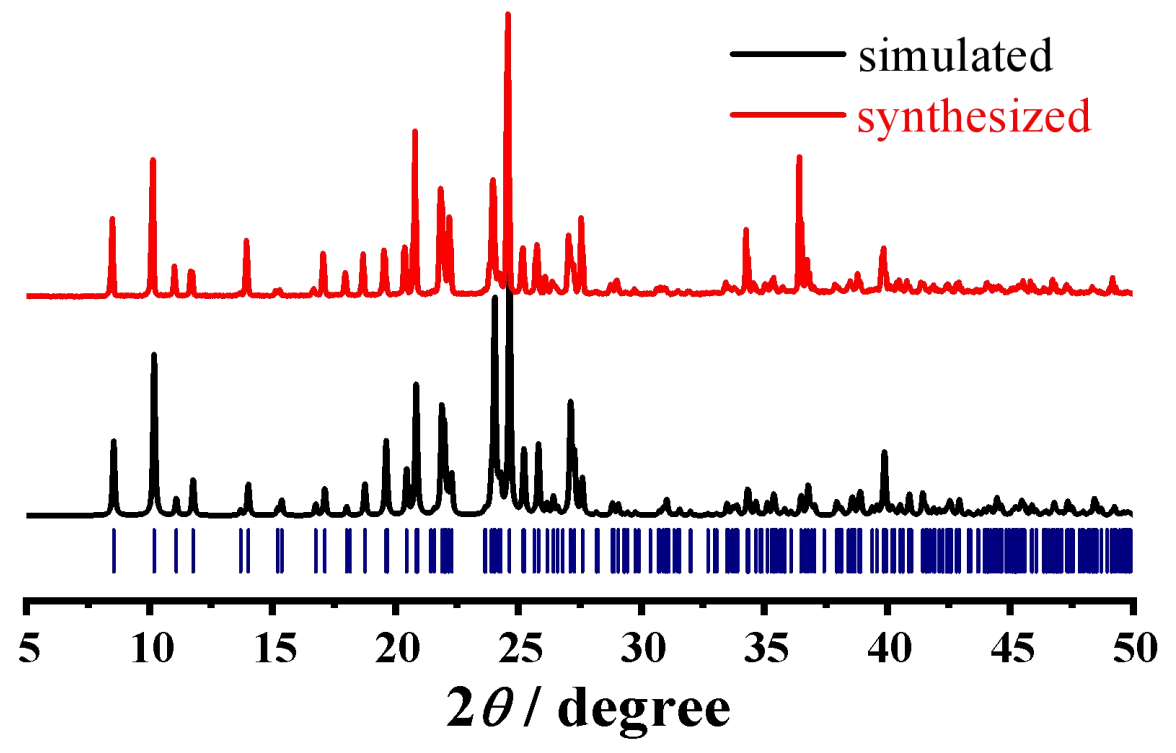

Figure S2. The PXRD patterns of 2. 


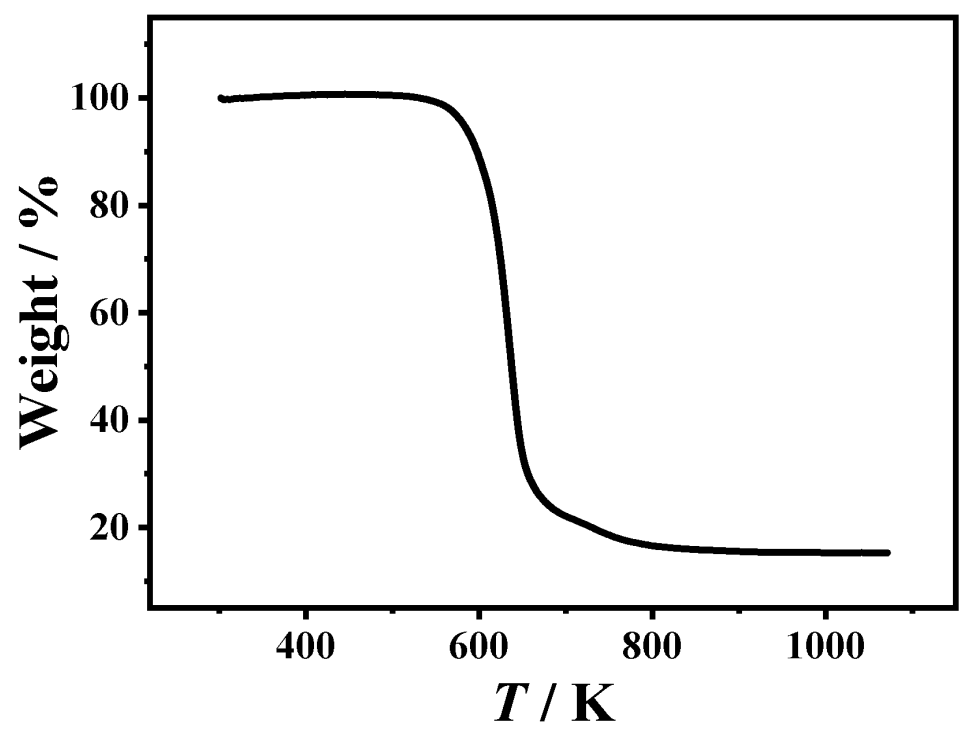

Figure S3. TG profile of $\mathbf{1}$.

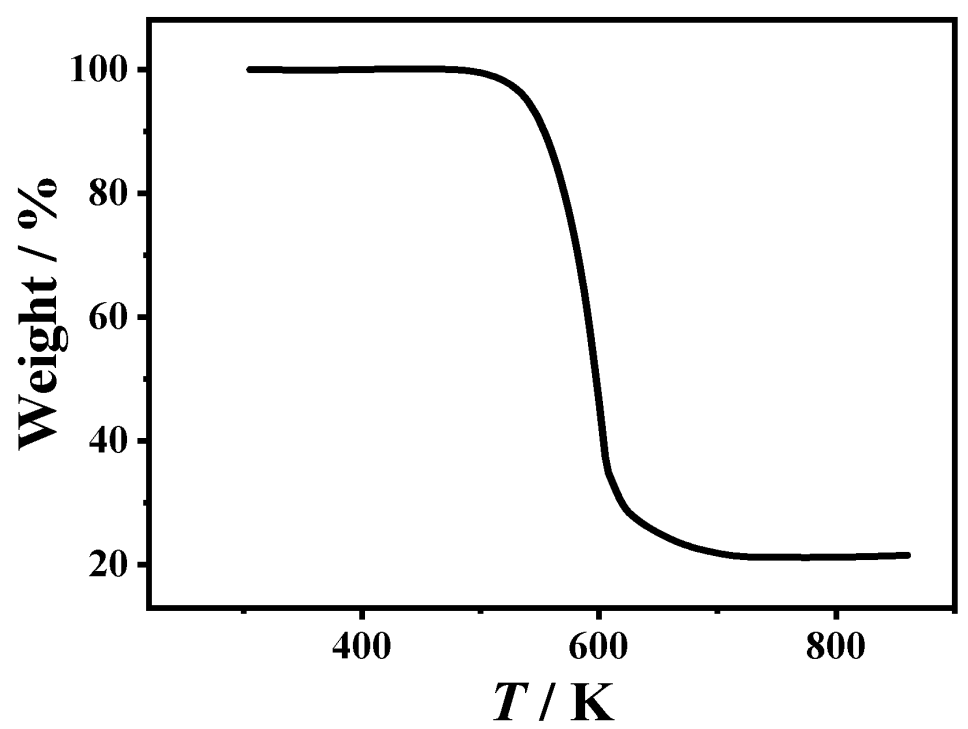

Figure S4. TG profile of 2. 


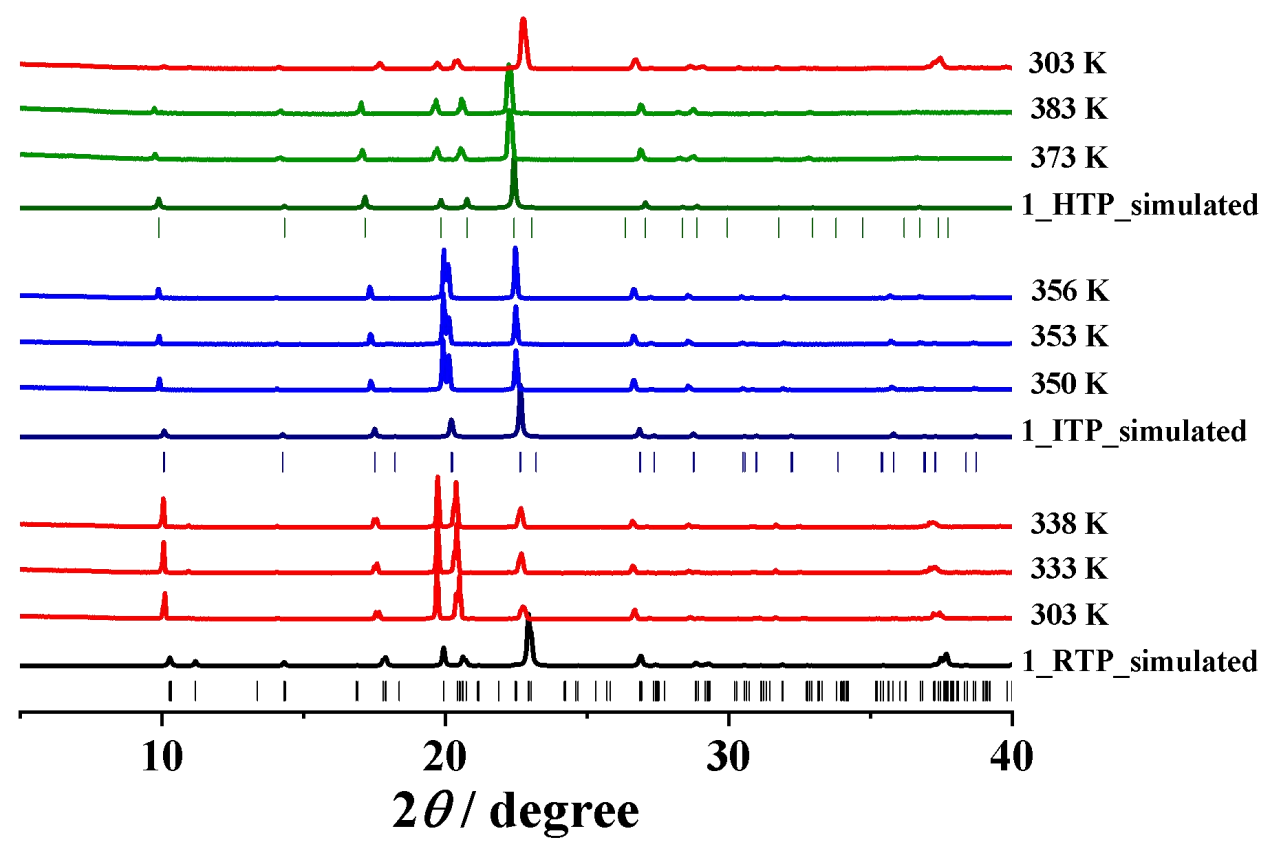

Figure S5. The variable-temperature PXRD patterns of $\mathbf{1 .}$

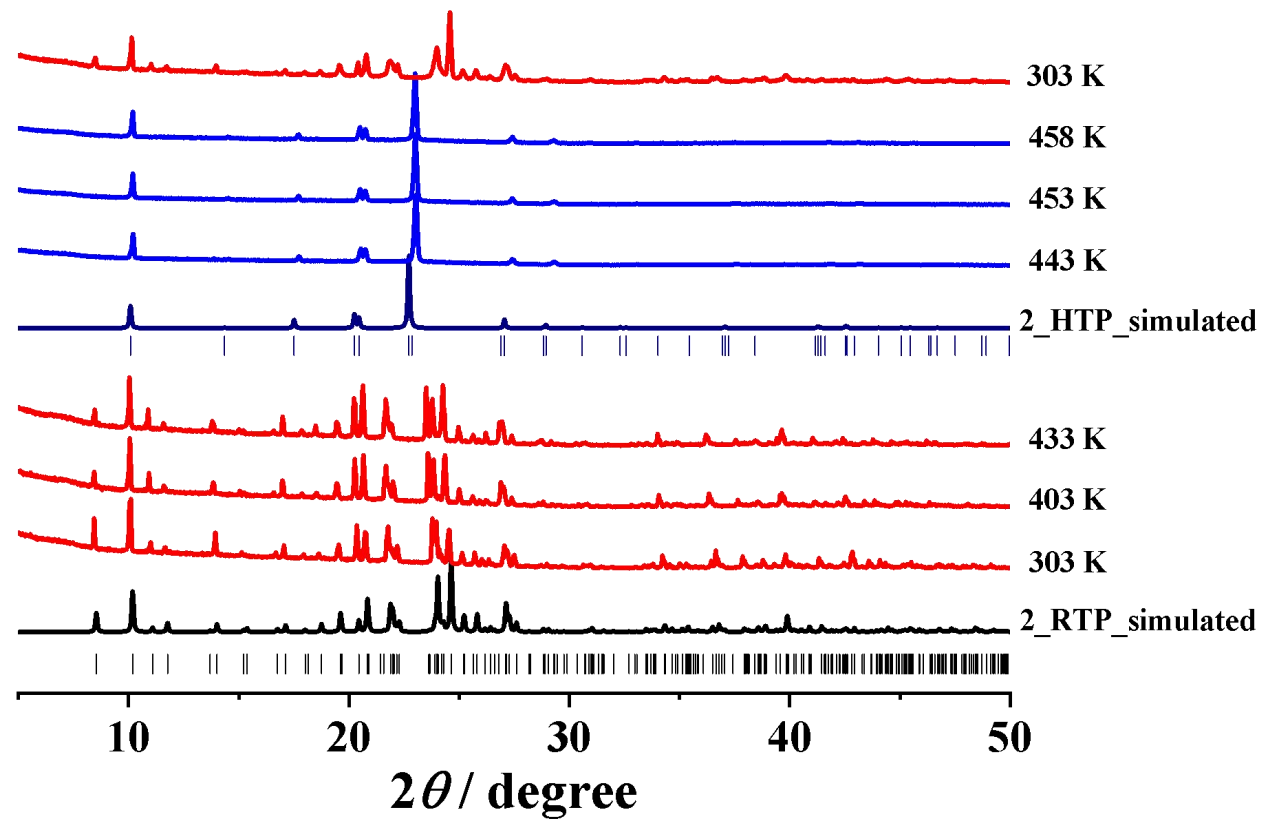

Figure S6. The variable-temperature PXRD patterns of 2. 


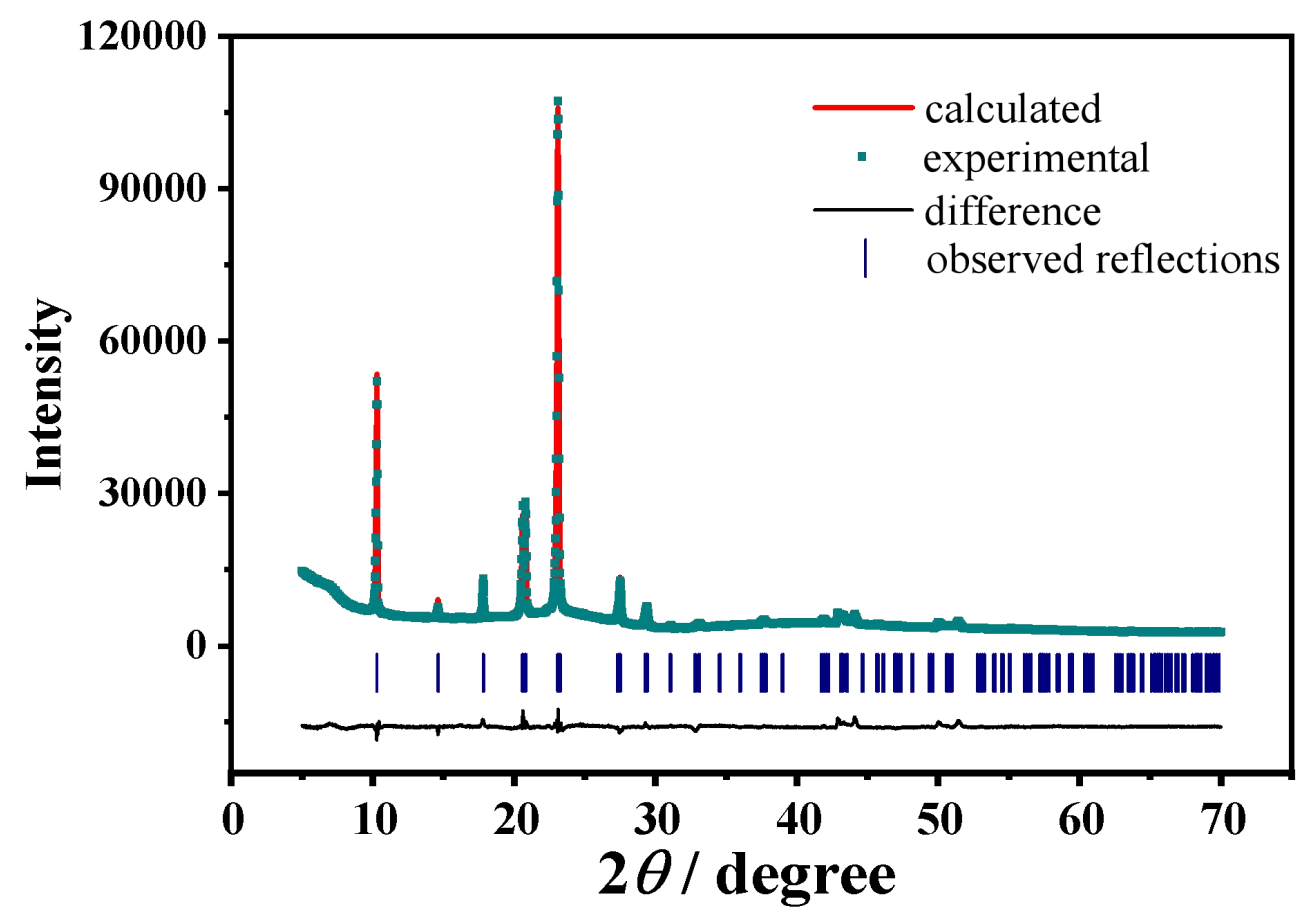

Figure S7. The final Rietveld refinement plot of structure in 2_HTP: calculated pattern (red solid line), experimental pattern (green dots), difference profile (bottom solid line), and peak positions (blue stick marks) allowed by the unit cell parameters and space group.

(a)

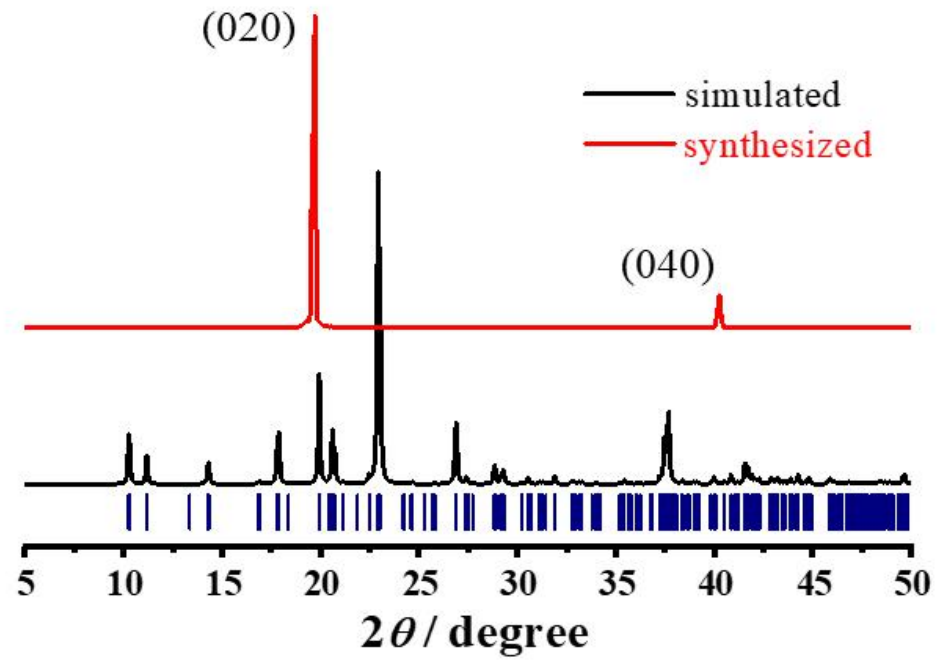

(b)

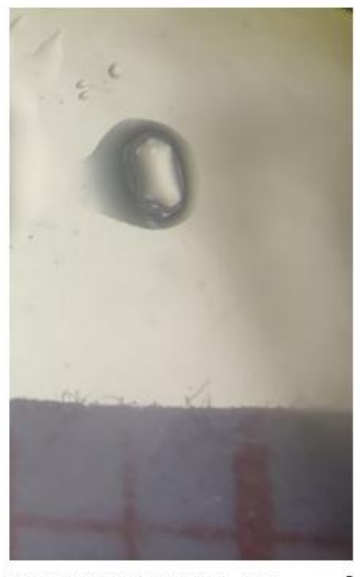

$0.67 \times 0.33 \times 0.25 \mathrm{~mm}^{3}$

Figure S8. The PXRD patterns at room temperature (a) and size (b) of sample of $\mathbf{1}$ for ferroelastic tests measured on the largest plane of single crystal for observation of ferroelastic domains, indicating the normal of the largest plane of the single crystal is the $b$ axis of 1_RTP and the $c$ axis of 1_ITP. 
(a)

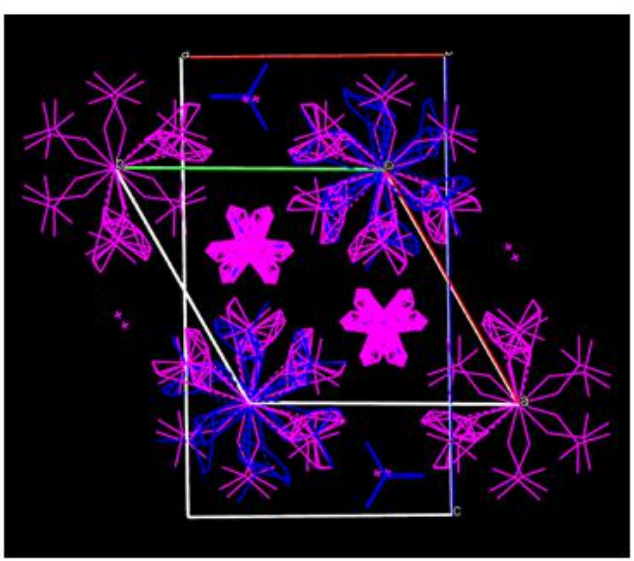

(b)

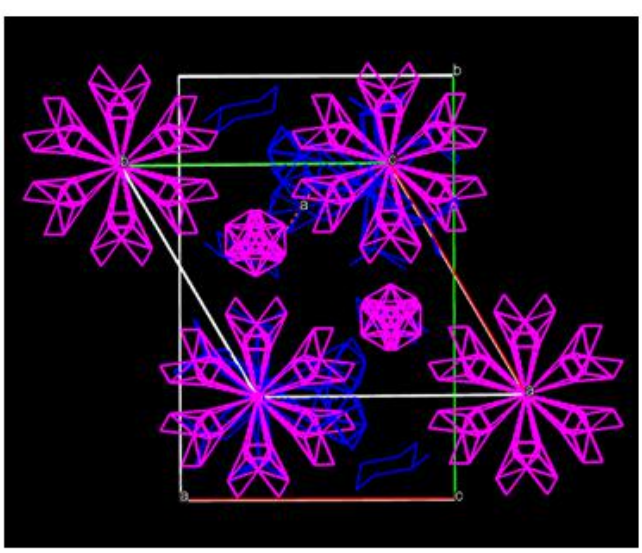

Figure S9. The cell transformation relationship (a) between 1_RTP (blue) and 1_ITP (magenta) parallel to the $c$ axis of 1_ITP; (b) between 2_RTP (blue) and 2_HTP (magenta) parallel to the $c$ axis of 2_HTP.

(a)

2_HTP

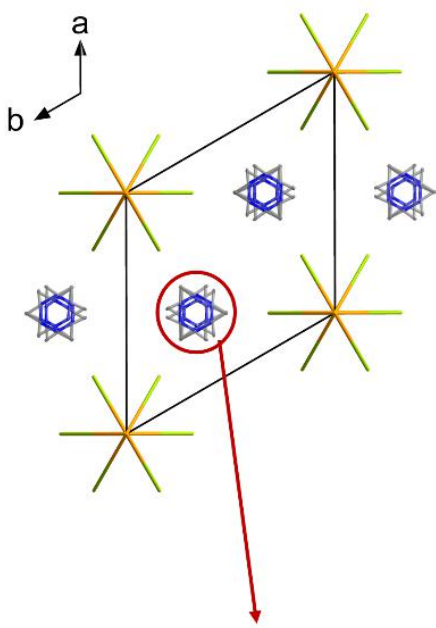

(c)

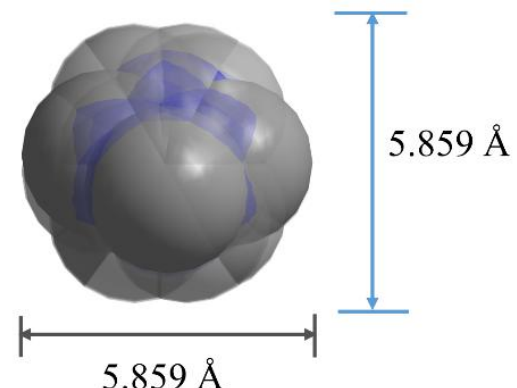

(b)

2_RTP
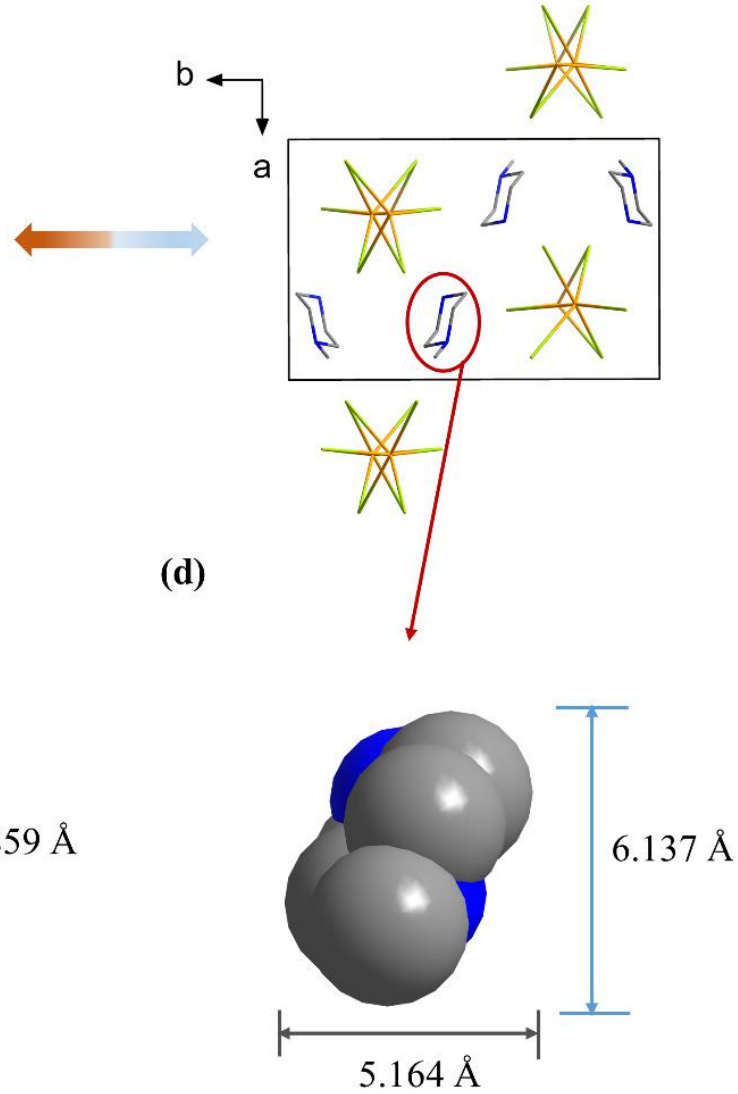

Figure S10. Crystal structures of 2_RTP (a) and 2_HTP (b) viewing along their $c$ axes. The size of $\mathrm{H}_{2} \mathrm{mpz}^{2+}$ cation along the different axes in 2_HTP (c) and 2_RTP (d). 
(a)

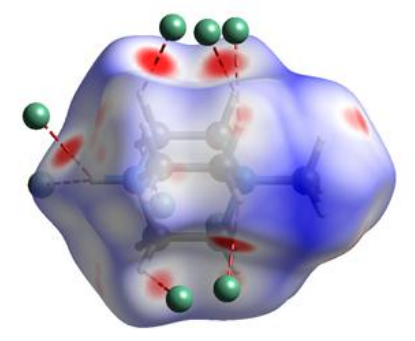

(e)

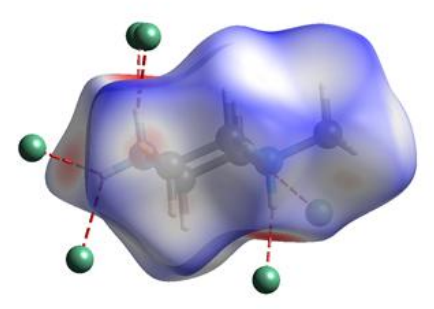

(b) all

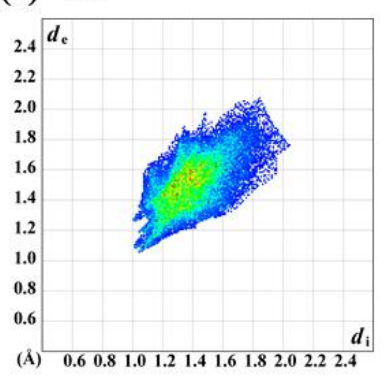

(f) all

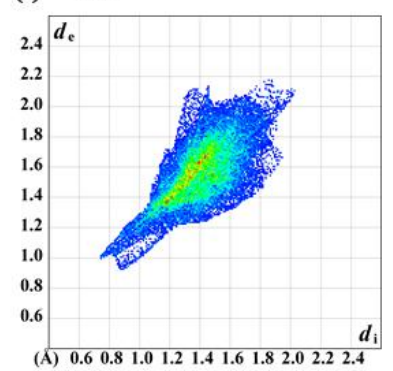

(c) $83.9 \%(\mathbf{H} \cdots \mathbf{F})$

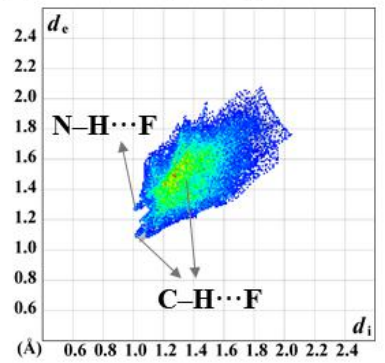

(g) $89.4 \%\left(H_{\cdots} \cdots \mathbf{F}\right)$

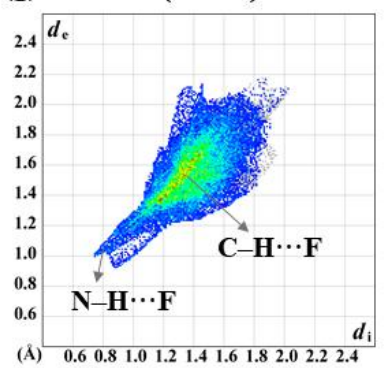

(d) $16.1 \%(\mathbf{H} \cdots \mathbf{H})$

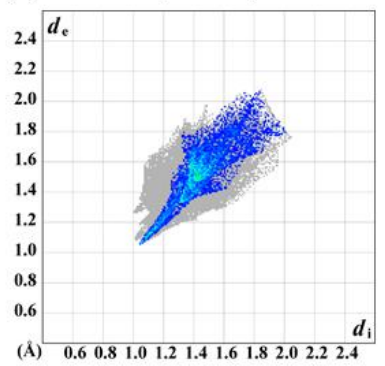

(h) $10.6 \%(\mathbf{H} \cdots \mathrm{H})$

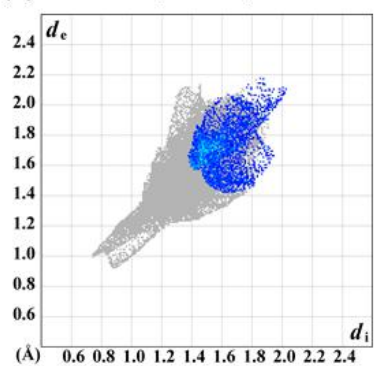

Figure S11. (a) Hirshfeld surfaces mapped with $d_{\text {norm }}$ over the range of -0.17 (red) to 1.10 (blue) for the Hmdabco ${ }^{2+}$ cation in 1_RTP (a) and -0.65 (red) to 1.10 (blue) for the $\mathrm{H}_{2} \mathrm{mpz}^{2+}$ cation in 2_RTP (e). Fingerprint plots for all contacts, $\mathrm{H} \cdots \mathrm{F}$ contacts and $\mathrm{H} \cdots \mathrm{H}$ contacts of guest cations in 1_RTP $(\mathrm{b}, \mathrm{c}, \mathrm{d})$ and 2_RTP $(\mathrm{f}, \mathrm{g}, \mathrm{h})$. 
Table S1. The variable-temperature unit-cell parameters of 1_RTP, 1_ITP, 1_HTP, 2_RTP, and 2_HTP. The parameters of

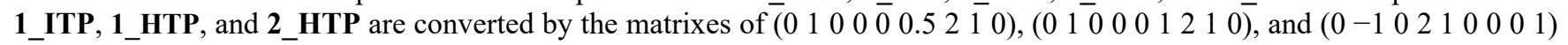
to match their corresponding ferroelastic phases, respectively.

\begin{tabular}{|c|c|c|c|c|c|c|c|}
\hline & $T / \mathrm{K}$ & $a / \AA$ & $b / \AA$ & $c / \AA$ & $V / \AA^{3}$ & $R_{\mathrm{p}} / \%$ & $R_{\mathrm{wp}} / \%$ \\
\hline \multirow{8}{*}{ 1_RTP } & 308 & $9.9340(7)$ & $8.9409(6)$ & $17.396(1)$ & 1545.1 & 4.08 & 5.13 \\
\hline & 313 & $9.9423(8)$ & $8.9385(7)$ & $17.403(1)$ & 1546.6 & 4.01 & 5.09 \\
\hline & 318 & $9.9510(9)$ & $8.9369(7)$ & 17.413(1) & 1548.5 & 4.06 & 5.38 \\
\hline & 323 & $9.9632(8)$ & $8.9357(7)$ & $17.428(2)$ & 1552.8 & 3.87 & 4.99 \\
\hline & 328 & $9.9751(10)$ & $8.9344(8)$ & $17.437(2)$ & 1554.0 & 4.09 & 5.27 \\
\hline & 333 & $9.9820(11)$ & $8.9334(10)$ & $17.451(2)$ & 1556.1 & 3.92 & 5.11 \\
\hline & 338 & $9.9927(12)$ & $8.9316(11)$ & $17.464(2)$ & 1558.7 & 3.94 & 4.99 \\
\hline & 343 & $9.9976(12)$ & $8.9308(11)$ & $17.461(2)$ & 1559.9 & 3.74 & 4.79 \\
\hline \multirow{4}{*}{ 1_ITP } & 350 & $10.105(1)$ & $8.8431(1)$ & $17.502(1)$ & 1563.9 & 4.66 & 6.36 \\
\hline & 352 & $10.110(1)$ & $8.7386(1)$ & $17.511(1)$ & 1564.8 & 4.45 & 5.93 \\
\hline & 355 & $10.114(1)$ & $8.8319(1)$ & $17.519(1)$ & 1564.9 & 4.12 & 5.80 \\
\hline & 357 & $10.117(1)$ & $8.8269(1)$ & $17.523(1)$ & 1564.9 & 4.42 & 5.91 \\
\hline \multirow{7}{*}{ 1_HTP } & 363 & $10.274(1)$ & $8.5918(7)$ & $17.795(1)$ & 1570.8 & 4.75 & 6.72 \\
\hline & 368 & $10.285(1)$ & $8.5832(7)$ & $17.815(1)$ & 1572.7 & 4.27 & 5.89 \\
\hline & 373 & $10.296(1)$ & $8.5757(7)$ & $17.833(1)$ & 1574.5 & 4.41 & 5.97 \\
\hline & 378 & $10.309(1)$ & $8.5682(7)$ & $17.856(1)$ & 1577.3 & 4.34 & 5.99 \\
\hline & 383 & $10.320(1)$ & $8.5607(7)$ & $17.874(1)$ & 1579.1 & 4.35 & 5.90 \\
\hline & 388 & $10.329(1)$ & $8.5547(7)$ & $17.890(1)$ & 1580.7 & 4.35 & 5.91 \\
\hline & 393 & $10.335(1)$ & $8.5528(7)$ & $17.900(1)$ & 1582.2 & 4.38 & 5.89 \\
\hline \multirow{8}{*}{ 2_RTP } & 398 & $10.535(1)$ & 16.201(1) & $8.6809(6)$ & 1468.0 & 4.73 & 6.55 \\
\hline & 403 & $10.535(1)$ & $16.209(1)$ & $8.6860(6)$ & 1469.7 & 4.49 & 6.05 \\
\hline & 408 & $10.535(1)$ & $16.219(1)$ & $8.6926(5)$ & 1471.8 & 4.15 & 5.48 \\
\hline & 413 & $10.533(1)$ & $16.228(1)$ & $8.6981(5)$ & 1473.4 & 4.08 & 5.37 \\
\hline & 418 & $10.534(1)$ & $16.241(1)$ & $8.7001(5)$ & 1475.0 & 4.02 & 5.35 \\
\hline & 423 & $10.533(1)$ & $16.256(1)$ & $8.7023(5)$ & 1476.7 & 4.09 & 5.43 \\
\hline & 428 & $10.531(1)$ & $16.273(1)$ & $8.7053(5)$ & 1478.8 & 4.31 & 5.86 \\
\hline & 433 & $10.530(1)$ & $16.293(1)$ & $8.7154(5)$ & 1482.2 & 3.46 & 4.69 \\
\hline \multirow{6}{*}{ 2_HTP } & 438 & $10.278(1)$ & $17.806(1)$ & $8.8158(5)$ & 1612.9 & 2.65 & 3.50 \\
\hline & 443 & $10.283(1)$ & $17.811(1)$ & $8.8131(6)$ & 1614.2 & 2.69 & 3.53 \\
\hline & 446 & $10.287(1)$ & $17.818(1)$ & $8.8121(5)$ & 1615.2 & 2.68 & 3.45 \\
\hline & 449 & $10.292(1)$ & $17.826(1)$ & $8.8113(5)$ & 1616.6 & 2.80 & 3.63 \\
\hline & 452 & $10.296(1)$ & $17.833(1)$ & $8.8109(6)$ & 1617.7 & 2.74 & 3.76 \\
\hline & 455 & $10.300(1)$ & $17.840(1)$ & $8.8100(6)$ & 1618.9 & 2.93 & 4.07 \\
\hline
\end{tabular}


Table S2. Thermal expansion coefficients of the principal axes in $\mathbf{1}$ and $\mathbf{2}$.

\begin{tabular}{|c|c|c|c|c|c|c|c|}
\hline & \multirow{2}{*}{$T / \mathrm{K}$} & \multirow{2}{*}{$\begin{array}{l}\text { Principle } \\
\text { axis }\end{array}$} & \multicolumn{3}{|l|}{ Directions } & \multirow{2}{*}{$\alpha / \mathrm{MK}^{-1}$} & \multirow{2}{*}{$\beta_{\mathrm{V}} / \mathrm{MK}^{-1}$} \\
\hline & & & $a$ & $b$ & $c$ & & \\
\hline \multirow[t]{3}{*}{ 1_RTP } & \multirow[t]{3}{*}{$308-343$} & $\mathrm{X}_{1}$ & 0.0000 & 1.0000 & 0.0000 & $-31(2)$ & \multirow[t]{3}{*}{$294(10)$} \\
\hline & & $\mathrm{X}_{2}$ & 0.5850 & 0.0000 & -0.8111 & $112(5)$ & \\
\hline & & $\mathrm{X}_{3}$ & -0.9727 & 0.0000 & -0.2319 & $210(6)$ & \\
\hline \multirow[t]{3}{*}{ 1_ITP } & \multirow[t]{3}{*}{$350-357$} & $X_{1}$ & 0.0000 & -1.0000 & 0.0000 & $-260(3)$ & \multirow[t]{3}{*}{$125(23)$} \\
\hline & & $X_{2}$ & -1.0000 & 0.0000 & 0.0000 & $172(13)$ & \\
\hline & & $\mathrm{X}_{3}$ & 0.0000 & 0.0000 & 1.0000 & $172(13)$ & \\
\hline \multirow[t]{3}{*}{ 1_HTP } & \multirow[t]{3}{*}{$363-393$} & $X_{1}$ & 0.0000 & 1.0000 & 0.0000 & $-157(11)$ & \multirow[t]{3}{*}{$249(7)$} \\
\hline & & $\mathrm{X}_{2}$ & -1.0000 & 0.0000 & 0.0000 & 203(8) & \\
\hline & & $\mathrm{X}_{3}$ & 0.0000 & 0.0000 & 1.0000 & 203(8) & \\
\hline \multirow[t]{3}{*}{ 2_RTP } & \multirow[t]{3}{*}{$398-433$} & $\mathrm{X}_{1}$ & 0.9735 & 0.0000 & -0.2288 & $-26(3)$ & \multirow[t]{3}{*}{$265(14)$} \\
\hline & & $\mathrm{X}_{2}$ & 0.2553 & 0.0000 & 0.9669 & 131(7) & \\
\hline & & $\mathrm{X}_{3}$ & 0.0000 & 1.0000 & 0.0000 & $155(8)$ & \\
\hline \multirow[t]{3}{*}{ 2_HTP } & \multirow[t]{3}{*}{$438-455$} & $X_{1}$ & 0.0000 & 0.0000 & 1.0000 & $-37(4)$ & \multirow[t]{3}{*}{$225(9)$} \\
\hline & & $\mathrm{X}_{2}$ & -1.0000 & 0.0000 & 0.0000 & $130(3)$ & \\
\hline & & $\mathrm{X}_{3}$ & 0.0000 & -1.0000 & 0.0000 & $130(3)$ & \\
\hline
\end{tabular}


Table S3. Linear fitting results of the variable-temperature unit-cell parameters of 1_RTP, 1_ITP and 1_HTP.

\begin{tabular}{|c|c|c|c|c|}
\hline & Fitting equations & $R^{2}$ & Deduced parameters at $T_{1}$, & Deduced parameters at $T_{1}$ \\
\hline \multirow{5}{*}{ 1_RTP } & $a / \AA=0.00191 T+9.3451$ & 0.9919 & $a=10.009 \AA$ & \\
\hline & $b / \AA=-0.000278 T+9.0257$ & 0.9830 & $b=8.9291 \AA$ & \\
\hline & $c / \AA=0.00226 T+16.698$ & 0.9922 & $c=17.484 \AA$ & \\
\hline & $\beta /^{\circ}=-0.00428 T+91.701$ & 0.9735 & $\beta=90.214^{\circ}$ & \\
\hline & & & $V=1562.5 \AA^{3}$ & \\
\hline \multirow{4}{*}{ 1_ITP } & $a / \AA=0.00174 T+9.4979$ & 0.9616 & $a=10.103 \AA$ & $a=10.126 \AA$ \\
\hline & $b / \AA=-0.00230 T+9.6492$ & 0.9993 & $b=8.8186 \AA$ & $b=8.8186 \AA$ \\
\hline & $c / \AA=0.00301 T+16.451$ & 0.9616 & $c=17.538 \AA$ & $c=17.538 \AA$ \\
\hline & & & $V=1566.1 \AA^{3}$ & $V=1566.1 \AA^{3}$ \\
\hline \multirow{4}{*}{ 1_HTP } & $a / \AA=0.00222 T+9.4673$ & 0.9961 & & $a=10.269 \AA$ \\
\hline & $b / \AA=-0.00149 T+9.1324$ & 0.9971 & & $b=8.5943 \AA$ \\
\hline & $c / \AA=0.00385 T+16.398$ & 0.9927 & & $c=17.788 \AA$ \\
\hline & & & & $V=1569.9 \AA^{3}$ \\
\hline
\end{tabular}

Table S4. Linear fitting results of the variable-temperature unit-cell parameters of 2_RTP and 2_HTP.

\begin{tabular}{llll}
\hline & Fitting equations & $R^{2}$ & Deduced parameters at $T_{2}$ \\
\hline & $a / \AA=-0.000147 T+10.594$ & 0.9305 & $a=10.531 \AA$ \\
2_RTP & $b / \AA=0.00259 T+15.162$ & 0.9737 & $b=16.284 \AA$ \\
& $c / \AA=0.000879 T+8.3325$ & 0.9547 & $c=8.713 \AA$ \\
& $\beta / /^{\circ}=-0.00557 T+100.005$ & 0.9502 & $\beta=97.593^{\circ}$ \\
& & & $V=1481.0 \AA^{3}$ \\
\hline \multirow{2}{*}{ 2HTP } & $a / \AA=0.00134 T+9.6918$ & 0.9964 & $a=10.272 \AA$ \\
& $b / \AA=0.00231 T+16.787$ & 0.9964 & $b=17.787 \AA$ \\
$c / \AA=-0.000323 T+8.9567$ & 0.9393 & $c=8.8168 \AA$ \\
& & $V=1609.3 \AA^{3}$ \\
\hline
\end{tabular}


Table S5. Hydrogen bonds in 1_RTP and 2_RTP.

\begin{tabular}{|c|c|c|c|c|c|c|}
\hline & D-H & $\mathrm{A}$ & $d(\mathrm{D}-\mathrm{H}) / \AA$ & $d(\mathrm{H}-\mathrm{A}) / \AA$ & $d(\mathrm{D}-\mathrm{A}) / \AA$ & $\mathrm{D}-\mathrm{H}-\mathrm{A} /{ }^{\circ}$ \\
\hline \multirow{15}{*}{ 1_RTP } & N2-H2 & F14 ${ }^{1}$ & 0.98 & 2.40 & $3.023(13)$ & 120.6 \\
\hline & N2-H2 & $\mathrm{F} 14 \mathrm{~A}^{1}$ & 0.98 & 2.46 & $3.135(15)$ & 125.4 \\
\hline & N2-H2 & F34 & 0.98 & 2.30 & $3.003(3)$ & 128.1 \\
\hline & $\mathrm{C} 1-\mathrm{H} 1 \mathrm{~A}$ & $\mathrm{~F} 33^{2}$ & 0.97 & 2.42 & $3.157(14)$ & 132.7 \\
\hline & C1-H1B & $\mathrm{F} 12 \mathrm{~A}^{3}$ & 0.97 & 2.50 & $3.213(7)$ & 130.6 \\
\hline & C1-H1B & F23A & 0.97 & 2.55 & $3.177(12)$ & 122.6 \\
\hline & $\mathrm{C} 2-\mathrm{H} 2 \mathrm{~B}$ & $\mathrm{~F} 31 \mathrm{~A}^{2}$ & 0.97 & 2.54 & $3.224(13)$ & 127.9 \\
\hline & C3-H3B & $\mathrm{F} 12 \mathrm{~A}^{3}$ & 0.97 & 2.33 & $3.092(6)$ & 134.7 \\
\hline & $\mathrm{C} 4-\mathrm{H} 4 \mathrm{~A}$ & $\mathrm{~F} 12^{3}$ & 0.97 & 2.35 & $3.134(7)$ & 137.4 \\
\hline & C5-H5A & $\mathrm{F} 22^{1}$ & 0.97 & 2.51 & $3.183(6)$ & 126.3 \\
\hline & C5-H5A & $\mathrm{F} 22 \mathrm{~A}^{1}$ & 0.97 & 2.29 & $3.129(15)$ & 144.3 \\
\hline & C5-H5B & $\mathrm{F} 13^{2}$ & 0.89 & 2.48 & $3.158(8)$ & 126.6 \\
\hline & C6-H6A & $\mathrm{F} 24^{2}$ & 0.97 & 2.56 & $3.198(4)$ & 123.2 \\
\hline & C6-H6A & $\mathrm{F} 31 \mathrm{~A}^{2}$ & 0.97 & 2.51 & $3.204(12)$ & 128.5 \\
\hline & C6-H6B & $\mathrm{F} 22^{1}$ & 0.97 & 2.53 & $3.192(5)$ & 125.8 \\
\hline \multirow{16}{*}{ 2_RTP } & $\mathrm{N} 2-\mathrm{H} 2 \mathrm{~A}$ & F33 & 0.89 & 2.09 & 2.792(7) & 134.5 \\
\hline & $\mathrm{N} 2-\mathrm{H} 2 \mathrm{~A}$ & $\mathrm{~F} 14^{4}$ & 0.89 & 2.21 & $2.817(9)$ & 125.2 \\
\hline & $\mathrm{N} 2-\mathrm{H} 2 \mathrm{~A}$ & $\mathrm{~F} 14 \mathrm{~A}^{4}$ & 0.89 & 2.43 & $3.069(9)$ & 129.4 \\
\hline & $\mathrm{N} 2-\mathrm{H} 2 \mathrm{~A}$ & F33A & 0.89 & 2.15 & $2.882(12)$ & 139.0 \\
\hline & N2-H2B & $\mathrm{F} 12^{5}$ & 0.89 & 2.33 & $3.055(9)$ & 139.1 \\
\hline & N2-H2B & $\mathrm{F} 12 \mathrm{~A}^{5}$ & 0.89 & 1.95 & $2.759(6)$ & 150.0 \\
\hline & N2-H2B & $\mathrm{F} 13 \mathrm{~B}^{5}$ & 0.89 & 1.92 & $2.80(2)$ & 169.4 \\
\hline & N1-H1 & $\mathrm{F} 22^{6}$ & 0.98 & 1.91 & $2.852(14)$ & 161.1 \\
\hline & N1-H1 & $\mathrm{F} 22 \mathrm{~A}^{6}$ & 0.98 & 1.77 & $2.741(17)$ & 168.6 \\
\hline & $\mathrm{C} 1-\mathrm{H} 1 \mathrm{~A}$ & $\mathrm{~F} 12^{5}$ & 0.97 & 2.49 & $3.221(7)$ & 131.7 \\
\hline & C1-H1A & $\mathrm{F} 12 \mathrm{~A}^{5}$ & 0.97 & 2.43 & $3.138(7)$ & 130.0 \\
\hline & $\mathrm{C} 2-\mathrm{H} 2 \mathrm{D}$ & $\mathrm{F} 24^{7}$ & 0.97 & 2.47 & $3.247(8)$ & 137.1 \\
\hline & $\mathrm{C} 2-\mathrm{H} 2 \mathrm{D}$ & $\mathrm{F} 24 \mathrm{~A}^{7}$ & 0.97 & 2.41 & $3.147(9)$ & 132.2 \\
\hline & $\mathrm{C} 3-\mathrm{H} 3 \mathrm{~A}$ & $\mathrm{~F}^{3} 3^{4}$ & 0.97 & 2.57 & $3.196(9)$ & 122.6 \\
\hline & C3-H3A & $\mathrm{F} 33 \mathrm{~A}^{4}$ & 0.97 & 2.41 & $3.068(14)$ & 124.8 \\
\hline & C5-H5A & $\mathrm{F} 12 \mathrm{~B}^{8}$ & 0.97 & 2.58 & $3.207(13)$ & 122.8 \\
\hline
\end{tabular}

Symmetry codes: $\left.{ }^{1)}-1+x,+y,+z ;{ }^{2)} 1-x, 1-y, 1-z ;{ }^{3)} 3 / 2-x,-1 / 2+y, 3 / 2-z ;{ }^{4)} 1-x, 1-y, 1-z ;{ }^{5)} 1-x, 1 / 2+y, 1 / 2-z ;{ }^{6}\right)+x, 1 / 2-y$, $-1 / 2+z ;{ }^{7)} 2-x, 1 / 2+y, 3 / 2-z ;{ }^{8)} 1-x, 1-y,-z$ 


\section{The calculation of the spontaneous strain}

For the present $-3 m \mathrm{~F} 2 / m$ species of $\mathbf{1}$, as the cell setting of trigonal phase is different from the

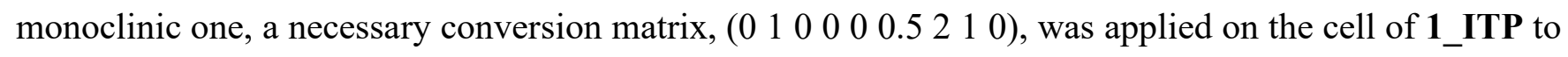
match with the cell of 1_RTP, giving a converted cell parameters for 1_ITP, as shown in Tables S1 and $\mathrm{S} 2$. For the present $6 / \mathrm{mmmF} 2 / m(s)$ species of $\mathbf{2}$, as the cell setting of hexagonal phase is different from

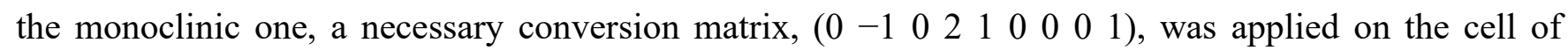
2_HTP to match with the cell of 2_RTP, giving a converted cell parameters for 2_RTP, as shown in Tables S1 and S3.

The spontaneous strain tensor is given as:

$$
\varepsilon_{S}=\left(\begin{array}{ccc}
\frac{a}{a_{0}}-1 & 0 & -\frac{c \cos \beta^{*}}{2 c_{0} \sin \beta_{0}^{*}} \\
0 & \frac{b}{b_{0}}-1 & 0 \\
-\frac{c \cos \beta^{*}}{2 c_{0} \sin \beta_{0}^{*}} & 0 & \frac{c \sin \beta^{*}}{c_{0} \sin \beta_{0}^{*}}-1
\end{array}\right) \quad \text { (equation 1) }
$$

In the equation, $a, b, c$, and $\beta^{*}$ refer to the low-symmetry form (1_RTP and 2_RTP), and $a_{0}, b_{0}, c_{0}$, and $\beta_{0} *$ are the converted monoclinic cell parameters of high-symmetry form (1_ITP and 1_RTP). $\beta$ and $\beta^{*}$ are reciprocal lattice angles.

According to the equation

$$
\varepsilon_{s}=\sqrt{\sum_{i, j} \varepsilon_{i j}^{2}}(i=1,2,3 ; j=1,2,3) \quad \text { (equation 2) }
$$

a total spontaneous strain for $\mathbf{1}$ and $\mathbf{2}$ was estimated by equations (1) and (2):

$$
\varepsilon_{s 1}=0.0133 \text { and } \varepsilon_{s 2}=0.129
$$

